Supporting Information.

\title{
Ultra-compact Iron Oxide Nanoparticles with a Monolayer Coating of Succinylated Heparin: A New Class of Renal-Clearable and Nontoxic T1 Agents for High-Field MRI
}

Manman Xie, ${ }^{\mathrm{a}}$ Ziyang Wang, ${ }^{\mathrm{a}}$ Qian Lu, ${ }^{\text {a }}$ Shuming Nie, ${ }^{\mathrm{b}}$ Christopher J. Butch ${ }^{\text {a }}$, Yiqing Wang ${ }^{* a}$ and Bo Dai ${ }^{* \mathrm{c}}$

a Department of Biomedical Engineering, College of Engineering and Applied Sciences,

State Key Laboratory of Analytical Chemistry for Life Science, Jiangsu Key Laboratory of

Artificial Functional Materials, Nanjing University, Nanjing 210093, China

${ }^{b}$ Department of Biomedical Engineering, University of Illinois at Urbana-Champaign, Urbana, IL 61801, United States

c Department of Cardio-Thoracic Surgery, Affiliated Drum Tower Hospital of Nanjing University Medical School, 321, Zhongshan Road, Nanjing 210008, China

*E-mail: chrisbutch@gmail.com, wangyiqing@nju.edu.cn and bodai1990@163.com 


\section{Supporting Information.}

Table S1. Literature data for commercially available contrast agents.

\begin{tabular}{|c|c|c|c|c|c|c|c|c|c|c|c|c|c|c|c|c|c|c|c|c|c|c|}
\hline \multirow{2}{*}{ Trade Name } & \multirow[b]{2}{*}{ Metal } & \multicolumn{4}{|c|}{$0.47 \mathrm{~T}$} & \multicolumn{4}{|c|}{$1.5 \mathrm{~T}$} & \multicolumn{4}{|c|}{$3 \mathrm{~T}$} & \multicolumn{4}{|c|}{$4.7 \mathrm{~T}$} & \multicolumn{4}{|c|}{$7 T$} & \multirow{2}{*}{\begin{tabular}{|l|} 
References \\
\end{tabular}} \\
\hline & & & r1 & & $\mathrm{r}^{2}$ & & $\mathrm{r} 1$ & & r2 & & $r 1$ & & r2 & & r1 & & r2 & & $\mathrm{r} 1$ & & r2 & \\
\hline Magnevist & $\mathrm{Gd}^{3+}$ & 3.4 & $(3.2-3.6)$ & 4 & (3.8-4.2) & 3.3 & $(3.1-3.5)$ & 3.9 & $(2.8-5.0)$ & 3.1 & $(2.8-3.4)$ & 3.7 & $(3.4-4.0)$ & 3.2 & $(3.0-3.4)$ & 4 & (3.8-4.2) & 3.02 & $(2.62-3.43)$ & 4.13 & (3.46-4.49) & $1,2,3$ \\
\hline Gadovist & $\mathrm{Gd}^{3+}$ & 3.7 & (3.5-3.9) & 5.1 & $(4.8-5.4)$ & 3.3 & $(3.1-3.5)$ & 3.9 & $(3.1-4.7)$ & 3.2 & $(2.9-3.5)$ & 3.9 & $(3.6-4.2)$ & 3.2 & $(3.0-3.4)$ & 3.9 & $(3.7-4.1)$ & 2.81 & $(2.65-3.49)$ & 3.75 & $(3.31-4.59)$ & $1,2,3$ \\
\hline Prohance & $\mathrm{Gd}^{3+}$ & 3.1 & $(2.9-3.3)$ & 3.7 & $(3.5-3.9)$ & 2.9 & $(2.7-3.1)$ & 3.2 & $(2.5-3.9)$ & 2.8 & $(2.6-3.0)$ & 3.4 & $(3.1-3.7)$ & 2.8 & $(2.7-2.9)$ & 3.7 & (3.5-3.9) & 2.69 & $(2.14-3.26)$ & 3.4 & $(3.04-4.07)$ & $1,2,3$ \\
\hline Multihance & $\mathrm{Gd}^{3+}$ & 4.2 & $(3.9-4.4)$ & 4.8 & $(4.6-5.0)$ & 4 & $(3.8-4.2)$ & 4.3 & $(3.8-4.8)$ & 4 & $(3.7-4.3)$ & 4.7 & $(4.4-5.0)$ & 4 & $(3.8-4.2)$ & 5 & $(4.7-5.3)$ & 3.63 & $(2.73-4.26)$ & 4.78 & $(3.94-5.63)$ & $1,2,3$ \\
\hline Dotarem & $\mathrm{Gd}^{3+}$ & 3.4 & $(3.2-3.6)$ & 4.1 & $(3.9-4.3)$ & 2.9 & $(2.7-3.1)$ & 3.2 & $(2.5-3.9)$ & 2.8 & $(2.6-3.0)$ & 3.3 & $(3.0-3.6)$ & 2.8 & $(2.7-2.9)$ & 3.7 & (3.5-3.9) & 2.73 & $(2.76-3.2)$ & 4.78 & $(3.19-4.08)$ & $1,2,3$ \\
\hline Omniscan & $\mathrm{Gd}^{3+}$ & 3.5 & (3.3-3.7) & 3.8 & $(3.6-4.0)$ & 3.3 & $(3.1-3.5)$ & 3.6 & $(3.0-4.2)$ & 3.2 & $(2.9-3.5)$ & 3.8 & $(3.5-4.1)$ & 3.3 & $(3.1-3.5)$ & 4.1 & $(3.9-4.3)$ & 2.87 & $(2.8-3.41)$ & 4.78 & $(3.09-4.41)$ & $1,2,3$ \\
\hline Teslascan & $\mathrm{Mn}^{2+}$ & 1.9 & $(1.8-2.0)$ & 2.1 & $(2.0-2.2)$ & 1.6 & $(1.5-1.7)$ & 2.1 & $(1.4-2.8)$ & 1.5 & $(1.3-1.7)$ & 2.3 & $(2.0-2.6)$ & 1.6 & $(1.5-1.7)$ & 2.7 & $(2.6-2.8)$ & 1.39 & $(1.17-1.6)$ & 3.38 & (2.6-4.15) & $1,2,3$ \\
\hline Optimark & $\mathrm{Gd}^{3+}$ & 4.2 & $(4.0-4.4)$ & 5.2 & $(4.9-5.5)$ & 3.8 & $(3.6-4.0)$ & 4.2 & $(3.5-4.9)$ & 3.6 & $(3.3-3.9)$ & 4.5 & $(4.2-4.8)$ & 3.8 & $(3.6-4.0)$ & 4.7 & (4.5-4.9) & -- & -- & -- & -- & 1 , \\
\hline Resovist & SPION $(80 \mathrm{~nm})$ & 20.6 & $(19.5-21.7)$ & 86 & $(82-90)$ & 8.7 & $(8.2-9.2)$ & 61 & $(54-68)$ & 4.6 & $(4.3-4.9)$ & 143 & $(132-154)$ & 2.8 & $(2.7-2.9)$ & 176( & (167-185) & 1.39 & $(0.8-1.97)$ & 53.6 & $(19.52-87.7)$ & $1,3,4$ \\
\hline Feridex & SPION $(50-180 \mathrm{~nm})$ & 27 & $(26-28)$ & 152 & $(144-160)$ & 4.7 & $(4.4-5.0)$ & 41 & $(39-43$ & 4.1 & (3.8-4.4) & 93 & $(87-99)$ & 2.3 & $(2.2-2.4)$ & 105 & $(100-110)$ & -- & -- & -- & -- & 1, \\
\hline Gadomer & $\mathrm{Gd}^{3+}(24)$ & 16.5 & $(15.7-17.3)$ & 17 & $(16-18)$ & 17.3 & $(16.4-18.2)$ & 22 & $(21-23)$ & 13 & $(12.3-13.7)$ & 23 & $(22-24)$ & 9.1 & $(8.6-9.6)$ & 22 & $(21-23)$ & -. & -. & -- & -- & 1, \\
\hline Vasovist & $\mathrm{Gd}^{3+}$ & 5.8 & $(5.5-6.1)$ & 6.7 & $(6.4-7.0)$ & 5.2 & $(4.9-5.5)$ & 5.9 & $(5.3-6.5)$ & 5.3 & $(5.0-5.6)$ & 6.1 & $(5.7-6.5)$ & 5.5 & $(5.2-5.8)$ & 6.9 & $(6.5-7.3)$ & -- & -- & -- & -- & 1, \\
\hline Primovist & $\mathrm{Gd}^{3+}$ & 5.3 & $(5.0-5.6)$ & 6.2 & $(5.9-6.5)$ & 4.7 & $(4.5-4.9)$ & 5.1 & $(4.5-5.7)$ & 4.3 & $(4.0-4.6)$ & 5.5 & $(5.2-5.8)$ & 4.9 & $(4.7-5.1)$ & 6.3 & $(6.0-6.6)$ & 5.17 & $(4.97-5.37)$ & 6.09 & $(5.17-7.01)$ & 1,3 \\
\hline Supravist & SPION (3-5nm) & 23.9 & $(22.7-25.1)$ & 54 & $(51-57)$ & 13.2 & $(12.5-13.9)$ & 44 & $(41-47)$ & 7.3 & $(6.9-7.7)$ & 57 & $(54-60)$ & 4.3 & $(4.1-4.5)$ & 66 & $(63-69)$ & .- & .- & .. & _- & 1,5 \\
\hline
\end{tabular}

References

\footnotetext{
$10.47,1.5,3$ and $4.7 T$ Rohrer, Martin, et al. "Comparison of magnetic properties of MRI contrast media solutions at different magnetic field strengths." Investigative radiology 40.11 (2005): $715-724$. data

$27 \mathrm{~T}$ data

Vignaud, Alexandre, et al. "Comparison of marketed Gadolinium-based Contrast Agents Relaxivities on Clinical MR scanner at 1.5 T, 3T and $7 \mathrm{~T}$ in water and plasma for a large range of physiological concentrations." Proc. Intl. Soc. Mag. Reson. Med. Vol. 22. 2014.

37 data Noebauer-Huhmann, I. M., et al. "MR contrast media at 7 Tesla-preliminary study on relaxivities." Proceedings 16 th Scientific Meeting, International Society for Magnetic Resonance in

4 Size $\quad$ Aguilar, Z. P. (2013). Nanopharmacology. Nanomaterials for Medical Applications, 293-360. doi:10.1016/b978-0-12-385089-8.00007-8

5 Size Thomsen, Henrik S. Contrast media. Ed. Judith AW Webb. Springer-Verlag Berlin Heidelberg, 2006.
} 


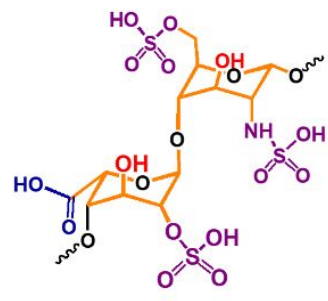

Heparin

(H)

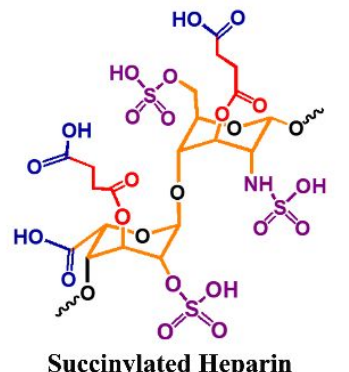

(SH)

When heparin is H5K, the resulted SH6k could coat the core of 6, 9 or $10 \mathrm{~nm}$ MIONs monolayer.

When heparin is H13k, the resulted SH15k coould coat the core of 14 or $18 \mathrm{~nm}$ MIONs monolayer.

Scheme S1. Synthesis schematic illustrations of SH coated MIONs with different core size.
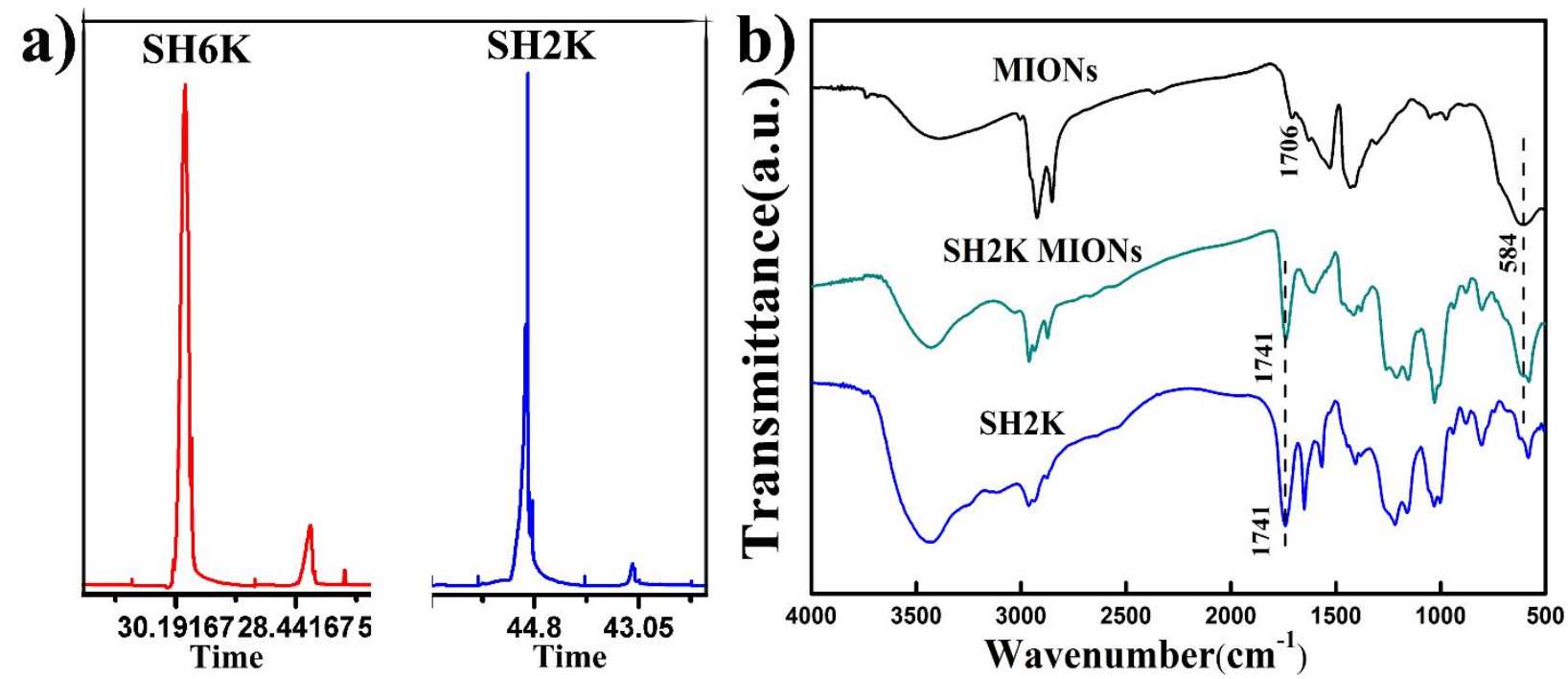

Figure S1. a) GPC chromatogram of SH6k and SH2k; b) FTIR spectra MIONs, SH2k MIONs and SH2k. 

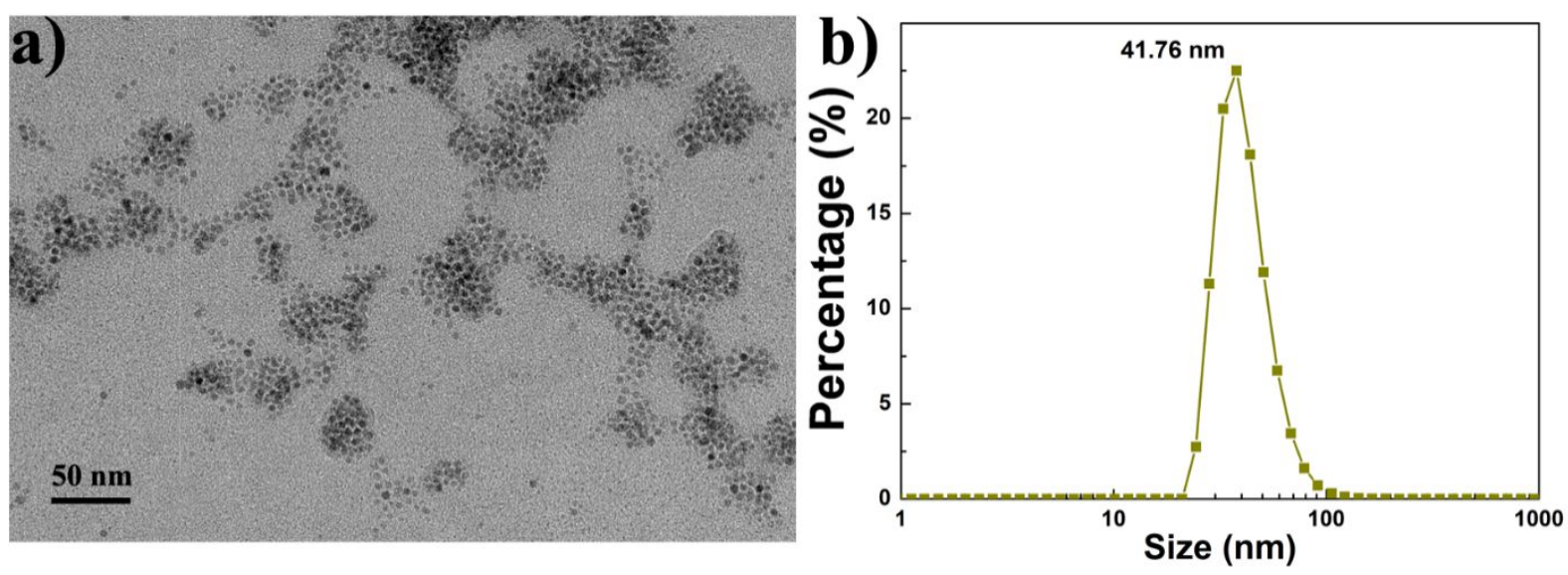

Figure S2. a) TEM and b) hydrodynamic size curves of 5nm SH15k MIONs.
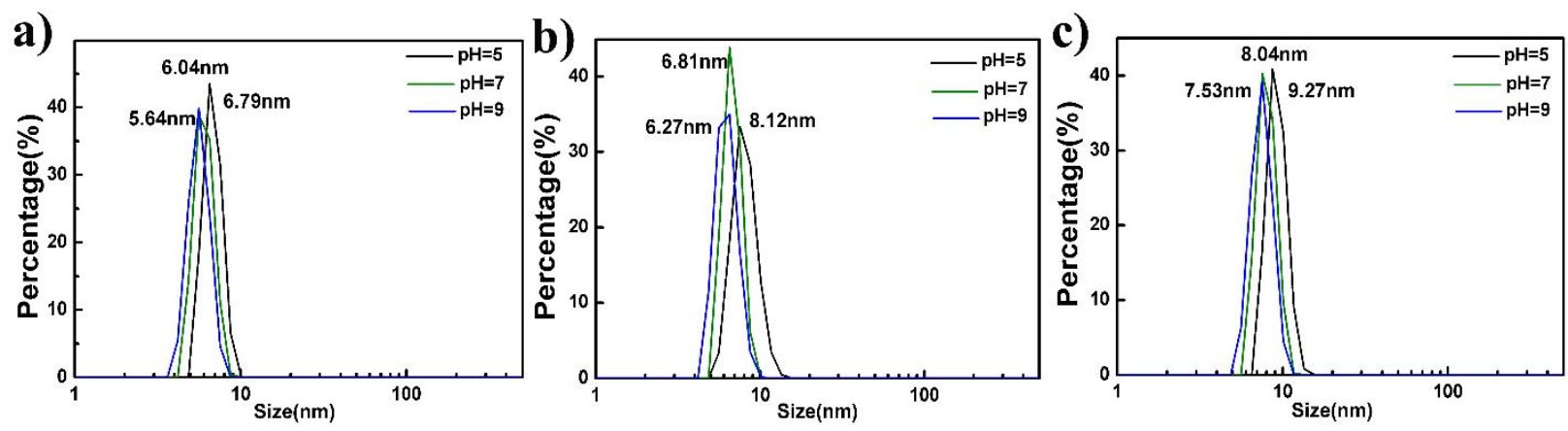

Figure S3. pH-dependent hydrodynamic size curves of a) $2 \mathrm{~nm}$, b) $3 \mathrm{~nm}$ and c) $5 \mathrm{~nm}$ SH2k MIONs. 


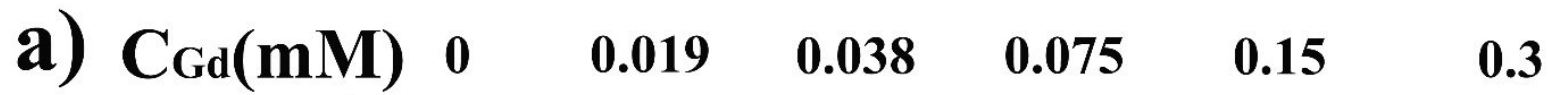
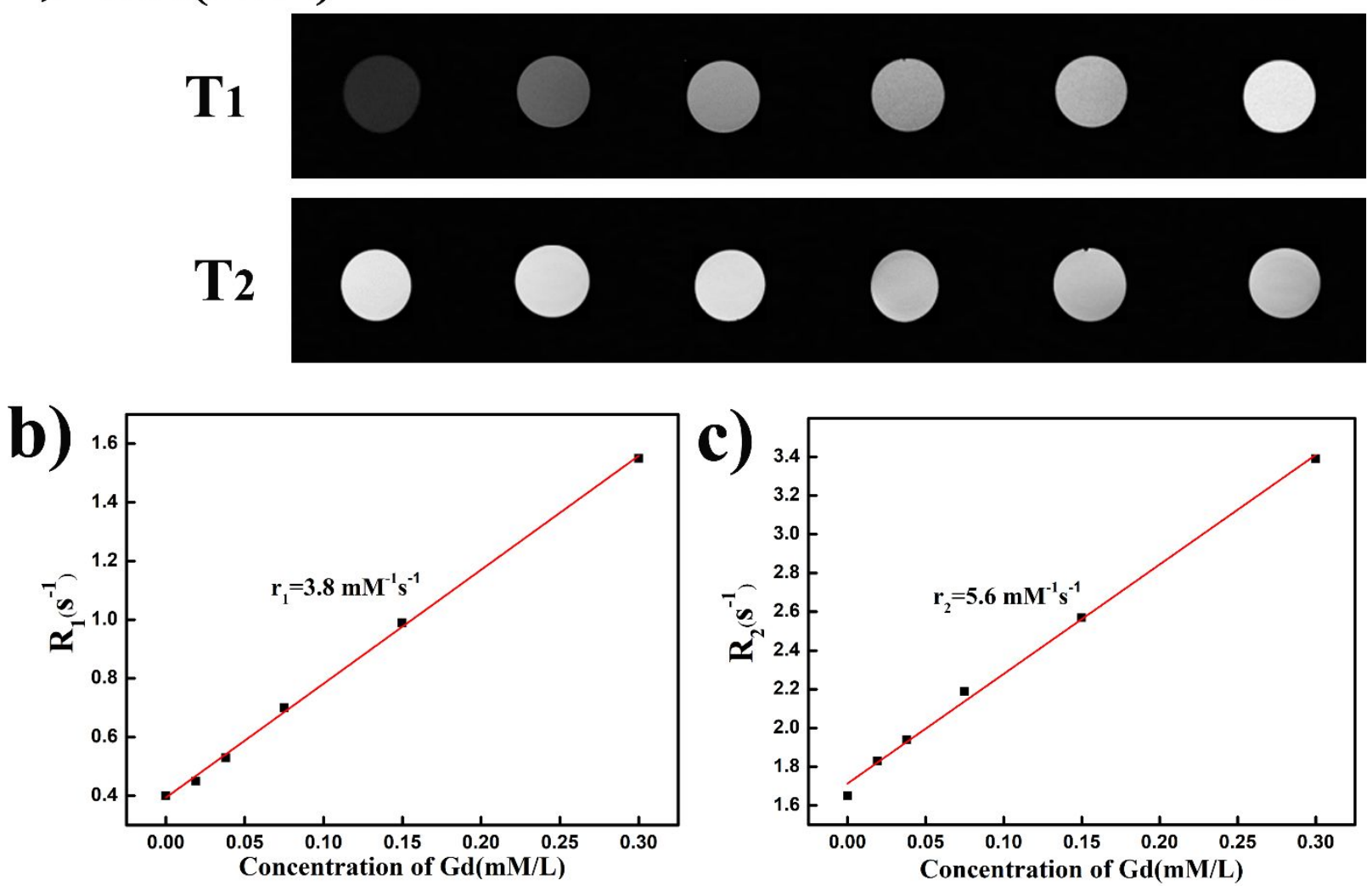

Figure S4. a) $T_{1}$ - and $T_{2}$-weighted MR images, b) $T_{1}$ relaxation rates $\left(r_{1}\right)$ and c) $T_{2}$ relaxation rates $\left(r_{2}\right)$ of GdDTPA with different Gd concentrations measured at $7 \mathrm{~T}$. 

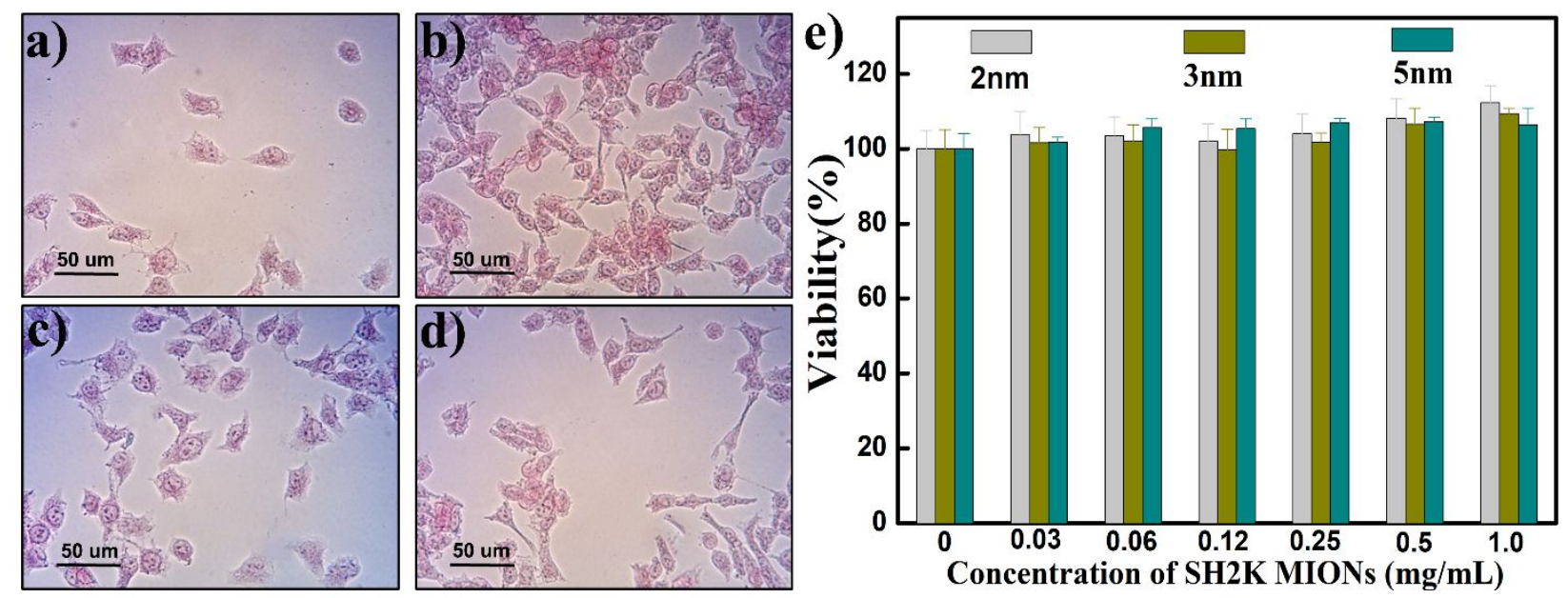

Figure S5. Prussian blue staining of KB incubated with a) control group, b) $2 \mathrm{~nm}$, c) $3 \mathrm{~nm}$ and d) $5 \mathrm{~nm} \mathrm{SH} 2 \mathrm{k}$ MIONs for $24 \mathrm{~h}$ at different Fe concentration. e) Cell viability assay results in the presence of SH2k MIONs on $\mathrm{KB}$ cells with various concentrations of $\mathrm{Fe}$ for $24 \mathrm{~h}$. The error bars represented \pm s.d. of six independent experiments. 


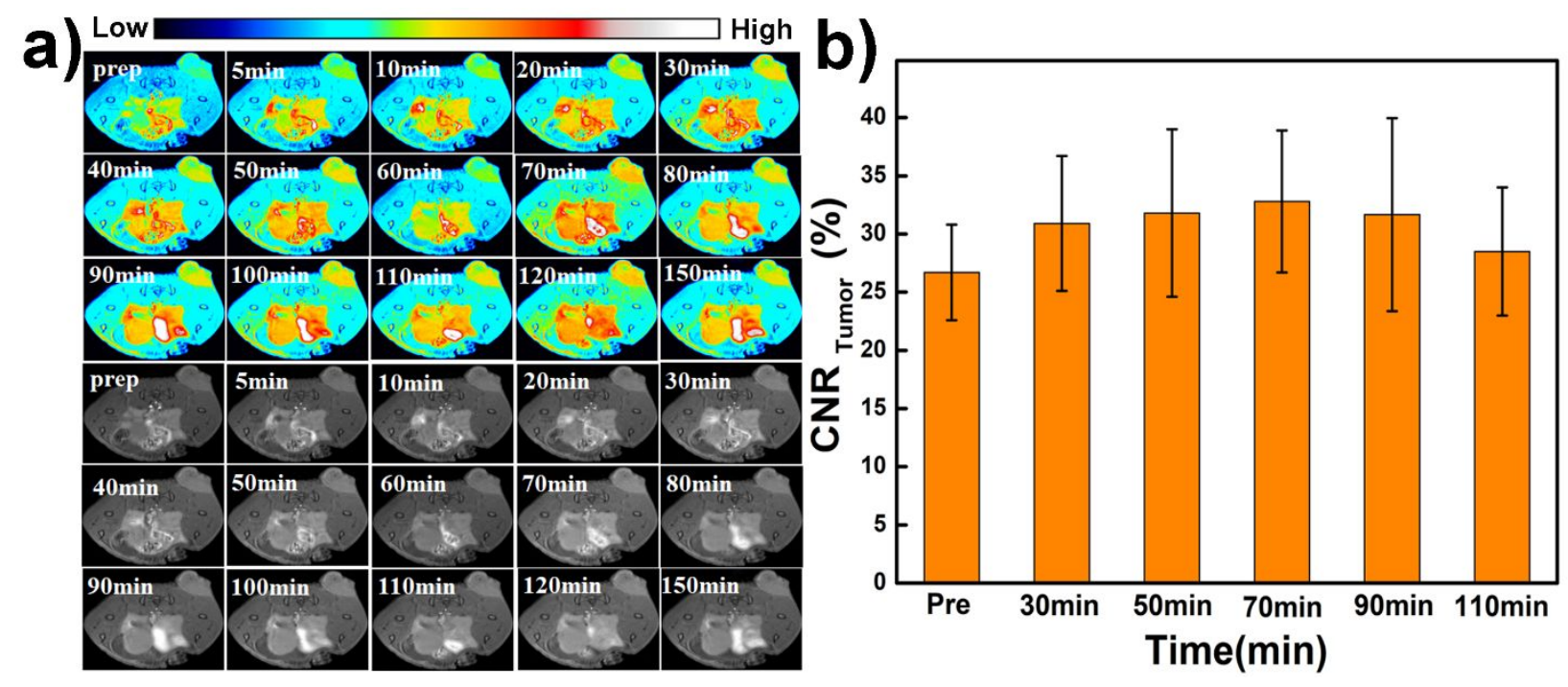

Figure S6. a) In vivo $T_{1}$-weighted MRI images of tumor and bladder in mice before and at different time points after the injection of $2 \mathrm{~nm} \mathrm{SH2k} \mathrm{MIONs;} \mathrm{b)} \mathrm{The} \mathrm{contrast-to-noise} \mathrm{ratio} \mathrm{(CNR)} \mathrm{of} \mathrm{tumor} \mathrm{to} \mathrm{normal} \mathrm{tissue.}$ 


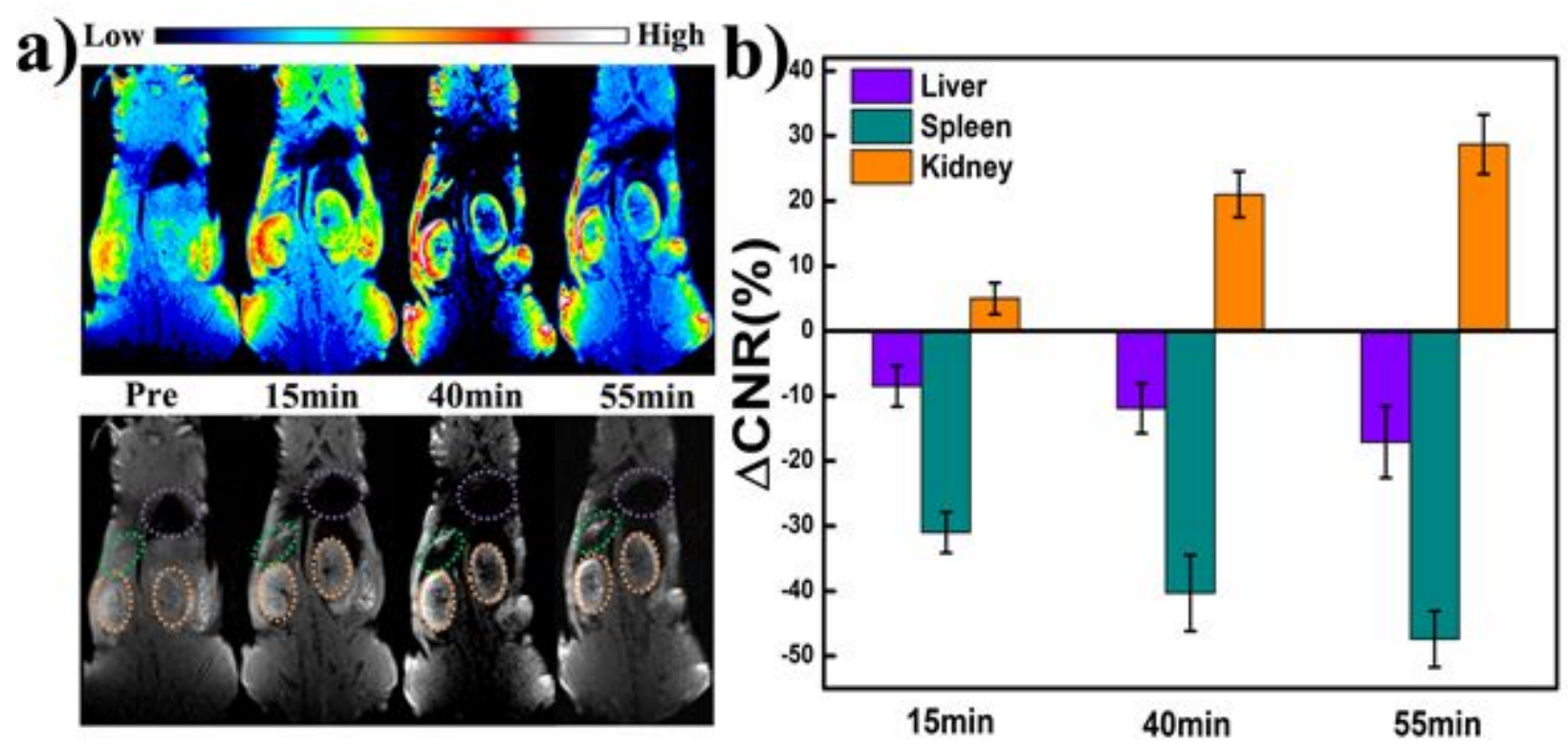

Figure S7. (a) In vivo $T_{1}$-weighted MRI images and (b) quantification of signal changes ( $\triangle \mathrm{CNR}$ ) of liver, spleen (orange dotted circles) and kidney (green dashed circles) in mice before and at different time points after the injection of $2 \mathrm{~nm} \mathrm{SH2k}$ MIONs. The changes in these signals suggest a general increase in background tissue signal due to the contrast agent injection, with preferential partitioning to the kidneys, (and per Figure 3, subsequently, the bladder) relative to the liver or spleen. While some particles are expected to undergo excretion via spleen and liver, the darkness of these organs relative to background, kidneys and spleen, suggests the contribution of this pathway is low. 

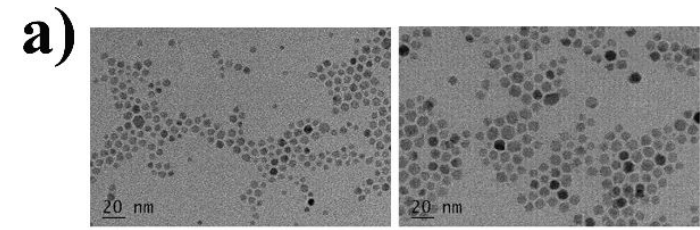

b)

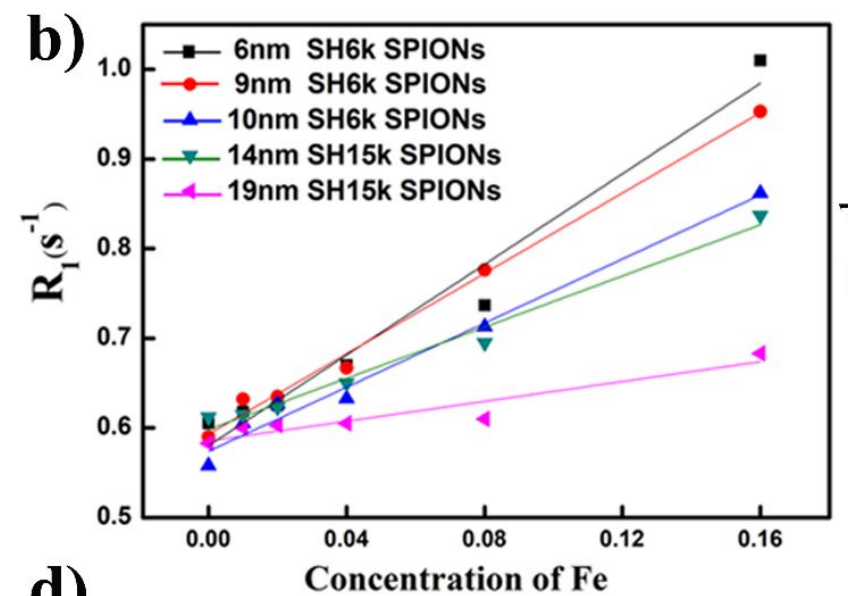

d)

\begin{tabular}{|l|c|c|c|c|c|}
\hline & $\begin{array}{c}6 \mathrm{~nm} \text { SH6k } \\
\text { MIONs }\end{array}$ & $\begin{array}{c}\text { 9nm SH6k } \\
\text { MIONs }\end{array}$ & $\begin{array}{c}10 \mathrm{~nm} \text { SH6k } \\
\text { MIONs }\end{array}$ & $\begin{array}{c}14 \mathrm{~nm} \text { SH15k } \\
\text { MIONs }\end{array}$ & $\begin{array}{c}18 \mathrm{~nm} \text { SH15k } \\
\text { MIONs }\end{array}$ \\
\hline $\mathrm{r}_{1}\left(\mathrm{mM}^{-1} \mathrm{~s}^{-1}\right)$ & 2.51 & 2.22 & 1.78 & 1.42 & 0.55 \\
\hline $\mathrm{r}_{2}\left(\mathrm{mM}^{-1} \mathrm{~s}^{-1}\right)$ & 124 & 172.2 & 180.6 & 203.9 & 217 \\
\hline
\end{tabular}

Figure S8. a) TEM images of $6 \mathrm{~nm}, 9 \mathrm{~nm}, 10 \mathrm{~nm}, 14 \mathrm{~nm}$ and $18 \mathrm{~nm}$ SH MIONs in water; b) $\mathrm{T}_{1}$ relaxation rates $\left(r_{1}\right)$, c) $T_{2}$ relaxation rates $\left(r_{2}\right)$ and d) relaxation properties of $6 \mathrm{~nm}$ SH6k, 9nm SH6k, 10nm SH6k, 14nm SH15k and $18 \mathrm{~nm}$ SH15k MIONs measured at $7 \mathrm{~T}$.

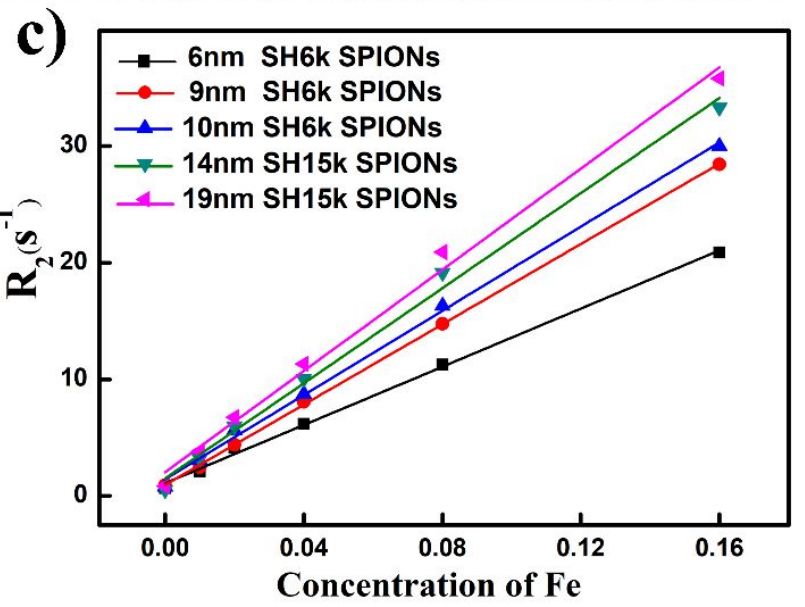

C) $\rightarrow-6 \mathrm{~nm}$ SH6k SPIONs

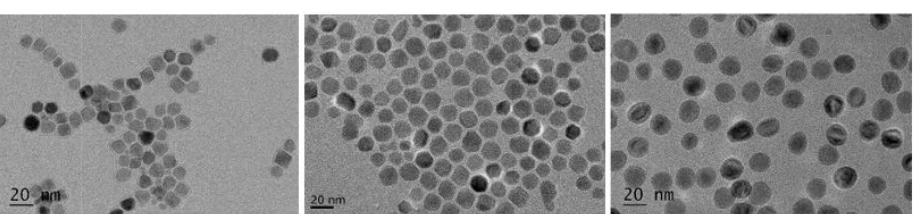

